\title{
Phenanthrene Tetraol
}

National Cancer Institute

\section{Source}

National Cancer Institute. Phenanthrene Tetraol. NCI Thesaurus. Code C107140.

A class of chemicals that are products of polycyclic aromatic hydrocarbon metabolism. 